\title{
Exploring alterations in hematological and biochemical parameters, enzyme activities and serum cortisol in Besnoitia besnoiti naturally infected dairy cattle
}

Luca Villa* ${ }^{*}$, Alessia Libera Gazzonis, Sergio Aurelio Zanzani, Silvia Mazzola, Alessia Giordano and Maria Teresa Manfredi

\begin{abstract}
Background: Besnoitia besnoiti is an Apicomplexan protozoa causative of bovine besnoitiosis, a chronic and debilitating disease of cattle, with a variety of pathological findings that could alter some laboratory parameters. A study was conducted in a bovine besnoitiosis endemically infected dairy herd located in Italy characterized by high intra-herd seroprevalence and cattle with clinical signs of the disease. In the study, alterations in laboratory parameters, i.e. hematological and biochemical parameters, enzyme activities and serum cortisol levels, in Besnoitia besnoiti naturally infected cows were investigated in depth.
\end{abstract}

Methods: Laboratory parameters in 107 cows, of which 61 were seronegative and 46 were seropositive to B. besnoiti, including 27 with clinical signs of bovine besnoitiosis, were compared. Generalized linear models were used to evaluate the effect of Besnoitia infection on the considered laboratory parameters.

Results: Hematological analyses revealed that B. besnoiti infection determined a significant alteration to the leukocyte differential, with a higher percentage of granulocytes and a lower percentage of lymphocytes in seropositive and clinically affected animals (Mann-Whitney U-test, $P=0.022$ ); erythrocyte and platelet counts did not show any difference between the considered groups of cows. Biochemistry tests evidenced that the parasite infection influenced serum protein values in seropositive cows and glutamate dehydrogenase values in clinically affected animals. No or only slight differences were revealed for all of the other biochemical and enzyme activity parameters in B. besnoitiinfected animals. In addition, despite the lack of statistical significance, seropositive and clinically affected cows evidenced higher concentrations of serum cortisol values compared to seronegative animals.

Conclusions: Although physiological, pathological and farm-related factors could have influenced the results in investigated animals, further studies involving more animals from different farms would be advisable to infer the role of $B$. besnoiti on these alterations, since laboratory parameters could help veterinarians in the diagnosis of bovine besnoitiosis in cattle.

Keywords: Bovine besnoitiosis, Dairy cattle, Hematology, Biochemistry, Enzyme activities, Cortisol

*Correspondence: luca.villa@unimi.it

Department of Veterinary Medicine, Università degli Studi di Milano, Via

dell'Università 6, 26900 Lodi, Italy

c) The Author(s) 2021. This article is licensed under a Creative Commons Attribution 4.0 International License, which permits use, sharing, adaptation, distribution and reproduction in any medium or format, as long as you give appropriate credit to the original author(s) and the source, provide a link to the Creative Commons licence, and indicate if changes were made. The images or other third party material in this article are included in the article's Creative Commons licence, unless indicated otherwise in a credit line to the material. If material is not included in the article's Creative Commons licence and your intended use is not permitted by statutory regulation or exceeds the permitted use, you will need to obtain permission directly from the copyright holder. To view a copy of this licence, visit http://creativeco mmons.org/licenses/by/4.0/. The Creative Commons Public Domain Dedication waiver (http://creativecommons.org/publicdomain/ zero/1.0/) applies to the data made available in this article, unless otherwise stated in a credit line to the data. 


\section{Background}

Bovine besnoitiosis is a parasitic disease caused by the cystogenic coccidian parasite Besnoitia besnoiti. In Europe, it is considered an emerging or re-emerging disease, with an increasing geographical distribution and the number of cases of infection [1]. In Italy, in the last decade, outbreaks of bovine besnoitiosis were reported, particularly in the northern regions [2-5].

Bovine besnoitiosis is a chronic and debilitating disease characterized by both cutaneous and systemic manifestations, compromising animal welfare and responsible for economic losses on affected farms $[1,6,7]$. The disease progresses in two sequential clinical phases. Initially, the intense multiplication of tachyzoites within endothelial cells causes vasculitis, hyperplasia, thrombosis, necrosis of venules and arterioles, and increased vascular permeability, subsequently resulting in congestion, hemorrhages and infarcts. The acute phase is thus characterized by hyperthermia, edemas and other non-specific clinical signs, including depression, swelling of the superficial lymph nodes, arrest of rumination, weight loss, anorexia, photophobia, epiphora, ocular and nasal discharge, tachycardia and tachypnea. The subsequent chronic phase is due to the slow replication of bradyzoites inside tissue cysts with tropism for connective tissues, surrounded by granulomatous inflammation. The skin becomes progressively thickened and wrinkled with alopecia, seborrhea, and hyperkeratosis, with the presence of pathognomonic subcutaneous thick-walled tissue cysts. Chronically infected cattle show loss of weight and progressive deterioration of the body condition $[6,8]$. The pathological findings occurring during both the acute and chronic phases of bovine besnoitiosis can lead to alterations in laboratory parameters.

A few studies focused on the hematological and biochemical changes during $B$. besnoiti infection. One study investigated both the impact of naturally acquired acute, subacute and chronic besnoitiosis on these parameters in three Simmental heifers and two Limousin cows and compared the changes in seronegative and seropositive Limousins from a German cow-calf operation, analyzing a high number of samples from a limited number of animals (224 samples from 9 Simmental and 75 samples from 54 Limousin cattle) [9]. Alterations in blood parameters of 11 beef cattle from an outbreak of bovine besnoitiosis in southwestern Spain [10] and in a naturally infected bull from South Africa were also reported [11]. Most of the studies concerned beef cattle, except that of Alshehabat et al. [12] in which selected metabolic biochemical parameters including enzyme activities associated with $B$. besnoiti infection were evaluated in dairy cattle from Jordan. Moreover, hematology and biochemistry changes were observed in goats affected by naturally acquired caprine besnoitiosis in Iran $[13,14]$.

To the best of our knowledge, no studies to date investigated the cortisol concentration in cows affected with bovine besnoitiosis. Cortisol is a cholesterol-derived steroid synthesized in the adrenal cortex under the control of the HPA (hypothalamic-pituitary-adrenal) axis that reacts to a wide range of stimuli from both internal and external origin. This glucocorticoid is the primary hormone involved in the stress response: its main action is to activate biological functions to respond to stress and to restore homeostasis after exposure to stressors. It is commonly used as an indicator of stress and welfare in animals [15]. Prolonged elevation of cortisol influences negatively the immune response, causing suppression of the immune system and increased susceptibility to disease; it also has adverse effects on the reproductive success of animals [16]. It was also reported that during immunosuppression $B$. besnoiti proliferation in cattle might be facilitated [17].

Considering that the spread of bovine besnoitiosis could be underestimated $[1,18]$ and that no studies to date focused on alterations in laboratory parameters in Holstein Friesian dairy cows under intensive farming conditions, the main goal of this study was to investigate alterations in hematological and biochemical parameters, including muscle and liver enzyme activity, and variations in serum cortisol levels in B. besnoiti naturally infected cows from an intensive dairy herd endemically affected by bovine besnoitiosis. Further aims were (i) to evaluate if the infection could be predicted by variations in the laboratory parameters investigated and (ii) to verify the effects of the parasite infection on health status and welfare of infected animals.

\section{Methods \\ Background}

The study was conducted in a bovine besnoitiosis endemically infected dairy herd hosting 217 Holstein Friesian cattle located in the province of Brescia (Lombardy, northern Italy). A serological screening for B. besnoiti was performed on the whole herd; Western Blot-positive results were considered as $B$. besnoiti seropositive animals [5]. In addition, a number of the seropositive animals were clinically examined to reveal symptoms and lesions of besnoitiosis. The herd resulted affected by high intra-herd B. besnoiti seroprevalence (23.5\% increasing up to $43.5 \%$ considering only cows) and a number $(n=27)$ of the seropositive animals suffered from clinical signs of the chronic phase of the disease; indeed, clinically affected cattle evidenced the presence of tissue cysts in at least one of the typical localizations (sclera, vulva, or skin). Moreover, a case of severe systemic besnoitiosis 
in a chronically affected cow was also recorded [5]. Besides, a form of generalized demodectic mange in two cows co-infected with B. besnoiti was also reported [19].

\section{Sample and data collection}

Overall, 107 cows from the infected herd, 46 seropositive to B. besnoiti and 61 seronegative, were included in the study. During the sampling for the serological screening [5], blood samples were also collected in tubes with EDTA for hematological analyses. In addition to the aliquot of the sera collected for serological analyses, a second aliquot was stored at $-20{ }^{\circ} \mathrm{C}$ for biochemistry, including liver and muscle enzyme activity analyses, and a third aliquot was stored at $-20{ }^{\circ} \mathrm{C}$ for cortisol determination. Epidemiological data, including individual data (breed, sex, productive category, age and origin) and reproductive parameters (number of parturitions and phase of lactation) were noted.

\section{Hematology}

Hematological analyses were performed on blood samples preserved in tubes with EDTA within $24 \mathrm{~h}$ from the collection time, using the Cell-Dyn 3500 analyzer (Abbott Laboratories, Abbott Park, IL, USA). The following hematological parameters were assessed: red blood cells $(\mathrm{RBC})$, hemoglobin $(\mathrm{Hb})$, hematocrit $(\mathrm{Ht})$, mean corpuscular volume (MCV), mean corpuscular hemoglobin $(\mathrm{MCH})$, mean corpuscular hemoglobin concentration (MCHC), red cell distribution width (RDW), white blood cells (WBC), lymphocytes, granulocytes, platelet count (PLT), mean platelet volume (MPV), plateletcrit (Pct) and platelet distribution width (PDW).

\section{Biochemistry and enzyme activity}

Biochemistry and enzyme activity analyses were carried out on serum using the automated analyzer BT3500 (Biotecnica Instruments S.p.a., Rome, Italy) using reagents, controls and calibrators provided by Futurlab $\mathrm{Srl}$ (Limena, Italy) and Randox Laboratories Ltd. (Crumlin Co., Antrium, UK; exclusively for BOHB and NEFA). The following analytes were measured (acronyms [as needed] followed by methods in parenthesis): albumin (bromochresol green), aspartate aminotransferase (AST, kinetic IFCC), $\beta$-hydroxybutyrate (BOHB, D-3-hydroxybutyrate dehydrogenase), calcium ( $\mathrm{Ca}$, orthocresoftaleine), magnesium (Mg, xylidyl blue), creatine kinase (CK, kinetic IFCC), glutamate dehydrogenase (GLDH, kinetic IFCC), lactate (LO-POD), lactate dehydrogenase (LDH, kinetic IFCC), non-esterified fatty acid (NEFA, ACS-ACOD), phosphorous (P, phosphomolibdate), total bilirubin (modified Jendrassik-Grof assay), total proteins (modified biuret). Globulin concentration was calculated by subtracting albumin concentration from total proteins, whereas the albumin:globulin ratio (A/G) was calculated by dividing albumin concentration by globulin concentration.

\section{Cortisol determination}

Cortisol concentration was assessed in serum samples using a commercially available enzyme-linked immunosorbent assay kit (Cortisol ELISA kit; article. CEA462Ge; Cloud Clone Corp., Katy, TX, USA). Sera stored at $-20{ }^{\circ} \mathrm{C}$ were analyzed after few days but always within 1 month of storage to avoid loss of bioactivity and contamination, according to the manufacturer's instructions. Each sample was prepared in duplicate, and absorbance was measured at a wavelength of $405 \mathrm{~nm}$, using a LabSystems Multiskan Ex microplate reader (Nepean, ON, Canada), according to the relevant standard curves. The mean recovery was $102.8 \% \pm 10.8$, The average intra- and inter-assay coefficients of variation were 5.7 and $7.6 \%$, respectively; the assay sensitivity was $5.15 \mathrm{ng} / \mathrm{ml}$. The laboratory technician was blinded to the hypotheses and conditions.

\section{Statistical analysis}

Values of hematology, biochemistry, enzyme activities and serum cortisol of seronegative and B. besnoiti seropositive dairy cattle were compared statistically. The same laboratory parameters were also compared between the groups of seronegative or asymptomatic seropositive cows and animals with clinical signs of chronic besnoitiosis. To assess normality, the Shapiro-Wilk test was used. To compare the two groups, the non-parametric MannWhitney U-test was performed. The level of significance was set at $P$ values of $<0.05$.

Separate generalized linear models (GLMs) with linear distribution were performed for each analyte to verify the influence of $B$. besnoiti infection on hematological and biochemical parameters, enzyme activities and serum cortisol levels (dependent variables). As predictor, the outcome of $B$. besnoiti infection was considered both as (i) seropositivity for B. besnoiti (dichotomous variable: presence $v s$ absence of antibodies based on Western Blot results) and (ii) clinical signs of bovine besnoitiosis (dichotomous variable: seronegative or asymptomatic seropositive animals vs clinically affected cows); these outcomes were evaluated individually, entered as independent variables in separate models. For the models containing the variable "seropositivity for B. besnoiti," all examined animals were included. For the models containing the variable "clinical signs of bovine besnoitiosis," 14 seropositive animals were excluded from the analysis, since it was not possible to perform a clinical examination and thus ascertain their clinical status. In addition, 
to consider the influence of the physiological status of the cows on the considered laboratory parameters, number of parturitions (dichotomous variable: primiparous $v s$ multiparous cows) and phase of lactation (ordinal variable: early, mid, late and dry) were also entered in each model as independent variables. In addition, the two-way interactions between $B$. besnoiti infection (seropositivity for B. besnoiti or clinical signs of bovine besnoitiosis), number of parturitions and phase of lactation were considered, to verify the overall influence of both the parasite infection and the physiological status of the cows on the considered laboratory parameters. All variables and their two-way interactions were entered in multivariate models developed through a backward selection procedure (significance level to remove variables from the model $=0.05$ ), based on Akaike's information criterion (AIC) values. When retained in the final models, the estimated means of the variable "phase of lactation" and the interactions were compared through pairwise comparisons. Statistical analysis was performed using IBM SPSS Statistics for Windows version 25.0 software (IBM Corp., Armonk, NY, USA).

\section{Results}

In this study, laboratory parameters of 107 cows, 61 of which were seronegative and 46 seropositive for $B$. besnoiti, including 27 with clinical signs of chronic bovine besnoitiosis, were investigated.

All sampled animals were female Holstein Friesian cows and all were born in the farm. The mean age of sampled cows was 46.8 months (standard deviation [SD] 17.7, minimum-maximum [Min-Max] 25.4-115.6 months). Forty-six of the cows were primiparous, with the remaining having had between two and six parturitions. Ninetyfive animals were lactating at the time of sampling: 31 of them were in the early, 37 in the mid and 27 in the late phase of lactation, respectively; the remaining 12 animals were in the dry phase.

The results of hematological and biochemical parameters, enzyme activities and serum cortisol levels are summarized in Table 1. Descriptive statistics were assessed according to the groups of seronegative and seropositive cows based on the Western Blot results for B. besnoiti; in addition, among seropositive animals, a subgroup of cows presenting clinical signs of bovine besnoitiosis was considered. The laboratory parameters results were also assessed according to the number of parturitions (primiparous and multiparous cows) and the phase of lactation (early, mid, late and dry), as reported in Additional file 1: Table S1 and Additional file 2: Table S2, respectively. For all of the considered laboratory parameters, specific reference ranges for dairy cows were used.

\section{Hematology}

Mean values for $\mathrm{RBC}, \mathrm{Hb}, \mathrm{Ht}$ and the erythrocyte indices (MCV, MCH, MCHC and RDW) were similar between seronegative, seropositive and clinically affected cows (Table 1). However, considering overall both seronegative and $B$. besnoiti seropositive animals, alterations in some of these parameters were revealed. In particular, mean $\mathrm{RBC}\left(6.13 \pm 0.70 \times 10^{6} / \mu \mathrm{l}\right)$, Ht $(27.02 \pm 2.66 \%)$ and RDW (15.23 $\pm 0.90 \%)$ values were low and mean MCV $\left(44.35 \pm 3.66 \mu^{3}\right)$ and $\mathrm{MCH}(20.29 \pm 3.01 \mathrm{pg})$ values were high, but remained within the limits of the reference interval, respectively (Table 1 ).

WBC counts were also similar in the considered groups of animals (seronegative, seropositive and clinically affected cows), and mean values fell within the reference interval (Table 1). Nonetheless, the leukocyte differential was altered, with a higher percentage of granulocytes and a lower percentage value of lymphocytes in seropositive ( $54.22 \pm 18.53$ and $45.78 \pm 18.53 \%$, respectively) and clinically affected $(53.52 \pm 18.46$ and $46.48 \pm 18.46 \%$, respectively) cows, particularly in primiparous seropositive cows $(62.19 \pm 16.51$ and $37.81 \pm 16.51 \%$, respectively) and primiparous animals with clinical signs of besnoitiosis (56.77 \pm 23.61 and $43.23 \pm 23.61 \%$, respectively), in comparison to seronegative and multiparous ones (Additional file 1: Table S1). Indeed, while the values for seronegative animals were equally distributed in terms of the reference interval for the leukocyte differential, $69.6 \%$ of seropositive cows, increasing up to $87.5 \%$ if considering only primiparous cows, showed a mean concentration of lymphocytes and granulocytes below and above the reference range, respectively.

The mean PLT, MPV, Pct and PDW values were slightly lower in seropositive $\left(286.41 \pm 106.87 \times 10^{3} / \mu \mathrm{l}\right.$, $8.82 \pm 0.32 \mu^{3}, 0.25 \pm 0.09 \%$ and $11.66 \pm 1.05 \%$, respectively) and clinically affected cows $\left(286.74 \pm 111.46 \times 10^{3} / \mu \mathrm{l}\right.$, $8.80 \pm 0.30 \mu^{3}, 0.25 \pm 0.10 \%$ and $11.63 \pm 1.17 \%$, respectively) than in seronegative animals $\left(307.77 \pm 115.04 \times 10^{3} / \mu \mathrm{l}\right.$, $13.43 \pm 35.35 \mu^{3}, 0.28 \pm 0.09 \%$, and $13.39 \pm 13.00 \%$, respectively), but mean values for all groups of cows fell within the reference intervals (Table 1 ).

\section{Biochemistry and enzyme activity}

Regarding serum total protein concentration, the mean of total proteins $(8.57 \pm 1.09 \mathrm{~g} / \mathrm{dl})$ was elevated in all cows; albumin $(3.03 \pm 0.55 \mathrm{~g} / \mathrm{dl})$ and globulin $(5.54 \pm 1.27 \mathrm{~g} /$ $\mathrm{dl})$ concentrations were at the lower and upper limit of the range, respectively, with a slightly lower value of the $\mathrm{A} / \mathrm{G}$ ratio $(0.59 \pm 0.22)$. In addition, seropositive $(8.34 \pm 1.04 \mathrm{~g} / \mathrm{dl})$ and clinically affected $(8.28 \pm 1.06 \mathrm{~g} /$ dl) cows showed lower values of total protein than seronegative animals $(8.73 \pm 1.11 \mathrm{~g} / \mathrm{dl})$; globulin concentrations were lower in seropositive $(5.22 \pm 1.10 \mathrm{~g} / \mathrm{dl})$ and 
Table 1 Descriptive statistics of hematological, biochemical, enzyme activity analyses and cortisol determination, according to the serological and clinical status of cows in a dairy cattle herd endemically infected by bovine besnoitiosis

\begin{tabular}{|c|c|c|c|c|c|c|c|c|}
\hline \multirow[t]{3}{*}{ Parameter } & \multirow[t]{3}{*}{ Unit } & \multicolumn{2}{|l|}{ Seronegative $^{a}$} & \multicolumn{4}{|l|}{ Seropositive ${ }^{a}$} & \multirow[t]{3}{*}{ Range } \\
\hline & & \multirow[t]{2}{*}{ Mean (SD) } & \multirow[t]{2}{*}{ Min-Max } & \multicolumn{2}{|l|}{ Overall } & \multicolumn{2}{|c|}{ Clinically affected ${ }^{a}$} & \\
\hline & & & & Mean (SD) & Min-Max & Mean (SD) & Min-Max & \\
\hline \multicolumn{9}{|l|}{ Hematology } \\
\hline $\mathrm{RBC}$ & $\times 10^{6} / \mu \mathrm{l}$ & $6.11(0.73)$ & $4.67-8.53$ & $6.15(0.67)$ & $4.80-7.14$ & $6.07(0.66)$ & $4.80-7.02$ & $6-10$ \\
\hline $\mathrm{Hb}$ & $\mathrm{g} / \mathrm{dl}$ & $12.46(1.55)$ & $8.86-15.88$ & $12.18(1.75)$ & $3.81-14.89$ & $12.04(2.01)$ & $3.81-14.45$ & $8-12$ \\
\hline $\mathrm{Ht}$ & $\%$ & $26.77(2.60)$ & $20.70-31.80$ & $27.35(2.73)$ & $22.20-32.40$ & $26.69(2.30)$ & $22.20-30.80$ & $20-35$ \\
\hline MCV & $\mu^{3}$ & $44.08(3.97)$ & $32.00-56.00$ & $44.70(3.22)$ & $38.00-50.00$ & $44.30(3.45)$ & $39.00-50.00$ & $40-50$ \\
\hline $\mathrm{MCH}$ & pg & $20.57(2.91)$ & $14.74-27.48$ & $19.92(3.12)$ & $7.62-24.96$ & $19.92(3.46)$ & $7.62-24.96$ & $15-20$ \\
\hline $\mathrm{MCHC}$ & $\mathrm{g} / \mathrm{dl}$ & $46.74(5.62)$ & $35.95-62.35$ & $44.64(6.34)$ & $17.17-56.71$ & $45.08(7.17)$ & $17.17-56.71$ & $30-40$ \\
\hline RDW & $\%$ & $15.23(0.99)$ & $13.40-17.50$ & $15.22(0.79)$ & $13.80-16.90$ & $15.20(0.84)$ & $13.90-16.90$ & $15-17$ \\
\hline WBC & $\times 10^{3} / \mu \mathrm{l}$ & $9.35(6.31)$ & $2.70-48.10$ & $9.69(6.58)$ & $4.70-37.20$ & $9.70(6.33)$ & $4.70-35.80$ & $6-12$ \\
\hline Lymphocytes & $\%$ & $53.52(13.69)$ & $11.00-97.00$ & $45.78(18.53)^{b}$ & $12.00-88.00$ & $46.48(18.46)$ & $12.00-86.00$ & $55-85$ \\
\hline Granulocytes & $\%$ & 46.48 (13.69) & $3.00-89.00$ & $54.22(18.53)^{b}$ & $12.00-88.00$ & $53.52(18.46)$ & $14.00-88.00$ & $15-45$ \\
\hline PLT & $\times 10^{3} / \mu l$ & 307.77 (115.04) & $17.10-490.00$ & $286.41(106.87)$ & $90.00-557.00$ & $286.74(111.46)$ & $90.00-557.00$ & $200-800$ \\
\hline MPV & $\mu^{3}$ & $13.43(35.35)$ & $8.20-285.00$ & $8.82(0.32)$ & $8.10-9.50$ & $8.80(0.30)$ & $8.30-9.40$ & $5-9$ \\
\hline Pct & $\%$ & $0.28(0.09)$ & $0.07-0.43$ & $0.25(0.09)$ & $0.08-0.47$ & $0.25(0.10)$ & $0.08-0.47$ & $0.1-0.4$ \\
\hline PDW & $\%$ & $13.39(13.00)$ & $9.70-113.00$ & $11.66(1.05)$ & $9.70-14.20$ & $11.63(1.17)$ & $9.70-14.20$ & $10-14$ \\
\hline \multicolumn{9}{|l|}{ Biochemistry } \\
\hline Total proteins & $\mathrm{g} / \mathrm{dl}$ & $8.73(1.11)$ & $6.30-11.50$ & $8.34(1.04)$ & $5.60-10.50$ & $8.28(1.06)$ & $5.60-10.50$ & $5.9-7.7$ \\
\hline Albumin & $\mathrm{g} / \mathrm{dl}$ & $2.96(0.52)$ & $2.00-4.20$ & $3.12(0.60)$ & $2.00-4.70$ & $2.93(0.52)$ & $2.00-3.90$ & $2.7-4.3$ \\
\hline Globulin & $\mathrm{g} / \mathrm{dl}$ & $5.78(1.34)$ & $2.90-8.90$ & $5.22(1.10)$ & $3.20-7.70$ & $5.36(0.92)$ & $3.60-6.80$ & $1.6-5$ \\
\hline $\mathrm{A} / \mathrm{G}$ ratio & & $0.56(0.22)$ & $0.26-1.28$ & $0.63(0.21)$ & $0.31-1.19$ & $0.56(0.13)$ & $0.37-0.82$ & $0.7-1.5$ \\
\hline Total bilirubin & $\mathrm{mg} / \mathrm{dl}$ & $0.56(0.36)$ & $0.09-1.63$ & $0.62(0.84)$ & $0.13-5.26$ & $0.72(1.05)$ & $0.13-5.26$ & $0.1-0.3$ \\
\hline $\mathrm{Ca}$ & $\mathrm{mg} / \mathrm{dl}$ & $9.56(0.74)$ & $7.00-11.10$ & $9.54(0.72)$ & $7.10-10.80$ & $9.40(0.81)$ & $7.10-10.80$ & $7.9-10$ \\
\hline$P$ & $\mathrm{mg} / \mathrm{dl}$ & $6.43(1.21)$ & $3.90-9.70$ & $6.18(1.21)$ & $4.00-10.40$ & $6.13(1.35)$ & $4.00-10.40$ & $4.6-9$ \\
\hline $\mathrm{Mg}$ & $\mathrm{mg} / \mathrm{dl}$ & $2.14(0.35)$ & $1.38-3.10$ & $2.15(0.35)$ & $1.22-2.93$ & $2.17(0.36)$ & $1.22-2.65$ & $1.4-2.3$ \\
\hline NEFA & $\mathrm{mmol} / \mathrm{l}$ & $0.32(0.24)$ & $0.06-1.05$ & $0.34(0.20)$ & $0.09-0.76$ & $0.33(0.21)$ & $0.12-0.76$ & $0.4-0.8$ \\
\hline $\mathrm{BOHB}$ & $\mathrm{mmol} / \mathrm{l}$ & $0.64(0.24)$ & $0.29-1.54$ & $0.60(0.28)$ & $0.05-1.48$ & $0.65(0.31)$ & $0.05-1.48$ & $0.03-0.3$ \\
\hline Lactate & $\mathrm{mmol} / \mathrm{l}$ & $2.82(0.92)$ & $1.47-5.23$ & $2.81(0.94)$ & $1.10-5.39$ & $2.78(1.02)$ & $1.10-5.39$ & $<2$ \\
\hline \multicolumn{9}{|l|}{ Enzymes } \\
\hline AST & $\mathrm{U} / \mathrm{I}$ & $74.40(33.17)$ & $43.00-252.00$ & 74.07 (21.24) & 47.00-159.00 & $75.26(23.16)$ & $48.00-159.00$ & $48-100$ \\
\hline CK & $\mathrm{U} / \mathrm{I}$ & 303.58 (1278.29) & $28.00-9971.00$ & $173.76(332.28)$ & $37.00-2182.00$ & $145.59(161.06)$ & $39.00-782.00$ & $44-228$ \\
\hline $\mathrm{LDH}$ & $\mathrm{U} / \mathrm{I}$ & $870.42(261.21)$ & $434.00-2173.00$ & $866.44(253.36)$ & $464.00-2113.00$ & $904.44(289.32)$ & $535.00-2113.00$ & $500-1500$ \\
\hline GLDH & $U / l$ & $33.42(51.46)$ & $6.40-367.00$ & 31.49 (31.09) & $0.00-137.70$ & $36.69(36.10)$ & $0.00-137.70$ & $<20$ \\
\hline Cortisol & $\mathrm{ng} / \mathrm{ml}$ & $6.81(6.41)$ & $0.77-35.48$ & $9.11(9.51)$ & $0.56-43.86$ & $8.54(11.36)$ & $0.56-43.86$ & $<10$ \\
\hline
\end{tabular}

All hematology parameters are defined in Section Hematology; all biochemistry and enzymes parameters are defined in Section Biochemistry and enzyme activity of the Methods

Min-Max, Minimum-Maximum values; SD, standard deviation

a Serological status (seronegative or seropositive) was determined according to Western Blot results. Clinically affected cows refer to those animals showing clinical signs of the disease

${ }^{b}$ Laboratory parameters significantly $(P<0.05)$ different vs. seronegative cows (Mann-Whitney UTest) are highlighted in italics

clinically affected $(5.36 \pm 0.92 \mathrm{~g} / \mathrm{dl})$ animals than seronegative cows $(5.78 \pm 1.34 \mathrm{~g} / \mathrm{dl})$. Albumin $(3.12 \pm 0.60 \mathrm{~g} /$ $\mathrm{dl})$ and $\mathrm{A} / \mathrm{G}$ ratio $(0.63 \pm 0.21)$ values were higher in seropositive cows, but slightly lower in clinically affected animals $(2.93 \pm 0.52 \mathrm{~g} / \mathrm{dl}$ and $0.56 \pm 0.13)$, in comparison to seronegative ones $(2.96 \pm 0.52 \mathrm{~g} / \mathrm{dl}$ and $0.56 \pm 0.22$, respectively). Values of serum protein differed according to the lactation phase: in particular, total protein values $(9.13 \pm 1.02 \mathrm{~g} / \mathrm{dl})$ were highest in the late phase, albumin $(3.68 \pm 0.34 \mathrm{~g} / \mathrm{dl})$ and $\mathrm{A} / \mathrm{G}$ ratio $(0.87 \pm 0.26)$ were highest in dry cows, whereas globulin values were highest in the late phase of lactation $(6.14 \pm 1.27 \mathrm{~g} / \mathrm{dl})$ but lowest in dry animals $(4.59 \pm 1.35 \mathrm{~g} / \mathrm{dl})$. 
Total bilirubin was elevated over the range limit in all cows $(0.58 \pm 0.61 \mathrm{mg} / \mathrm{dl})$, with higher values in seropositive $(0.62 \pm 0.84 \mathrm{mg} / \mathrm{dl})$ and clinically affected animals $(0.72 \pm 1.05 \mathrm{mg} / \mathrm{dl})$ than in seronegative ones $(0.56 \pm 0.36 \mathrm{mg} / \mathrm{dl})$.

In terms of minerals, the mean concentration of $\mathrm{Ca}$, $\mathrm{P}$, and $\mathrm{Mg}$ were within the limits, with similar values in seropositive and seronegative cows (Table 1).

Mean NEFA, BOHB and lactate values were similar between the groups of cows according to $B$. besnoiti seropositivity and clinical signs (Table 1 ). However, considering mean concentrations in all animals, values of $\mathrm{BOHB}$ $(0.62 \pm 0.25 \mathrm{mmol} / \mathrm{l})$ and lactate $(2.82 \pm 0.93 \mathrm{mmol} / \mathrm{l})$ were above the reference range, while NEFA values $(0.33 \pm 0.22 \mathrm{mmol} / \mathrm{l})$ fell within the reference interval.

Regarding enzyme activities, AST and LDH values fell within the normal range and showed similar values in all groups of animals (Table 1), with only a slightly higher value in clinically affected animals $(75.26 \pm 23.16$ and $904.44 \pm 289.32 \mathrm{U} / \mathrm{l}$, respectively) compared with seronegative cows $(74.40 \pm 33.17$ and $870.42 \pm 261.21 \mathrm{U} / \mathrm{l})$, respectively. CK level was elevated over the upper limit in seronegative animals $(303.58 \pm 1278.29 \mathrm{U} / \mathrm{l})$, while the mean value was within the reference interval in seropositive cows $(173.76 \pm 332.28 \mathrm{U} / \mathrm{l})$. GLDH values were above the reference limit in all the three groups of cows $(32.58 \pm 43.65 \mathrm{U} / \mathrm{l})$, with a slight higher value in clinically affected animals $(36.69 \pm 36.10 \mathrm{U} / \mathrm{l})$.

\section{Cortisol determination}

Seropositive $(9.11 \pm 9.51 \mathrm{ng} / \mathrm{ml})$ and clinically affected $(8.54 \pm 11.36 \mathrm{ng} / \mathrm{ml}) \quad$ cows evidenced higher values of cortisol when compared to seronegative animals $(6.81 \pm 6.41 \mathrm{ng} / \mathrm{ml})$. Cortisol levels were generally higher in the early lactation phase $(9.13 \pm 12.37 \mathrm{ng} / \mathrm{ml})$ and in dry cows $(10.96 \pm 6.27 \mathrm{ng} / \mathrm{ml})$, compared to cows in the $\mathrm{mid}(5.62 \pm 4.87 \mathrm{ng} / \mathrm{ml})$ and late $(8.27 \pm 5.76 \mathrm{ng} / \mathrm{ml})$ phase of lactation. In contrast, mean values were similar in primiparous $(7.15 \pm 7.33 \mathrm{ng} / \mathrm{ml})$ and multiparous $(8.04 \pm 8.52 \mathrm{ng} / \mathrm{ml})$ cows. However, overall mean cortisol levels $(7.92 \pm 8.09 \mathrm{ng} / \mathrm{ml})$ were generally elevated in all animals with respect to the recommended reference value $(5-10 \mathrm{ng} / \mathrm{ml})$.

\section{Statistical analysis}

Values of hematology, biochemistry, enzyme activities and serum cortisol were compared statistically using the non-parametric Mann-Whitney U-test considering the groups of seronegative versus B. besnoiti seropositive dairy cattle, and seronegative or asymptomatic seropositive versus clinically affected animals. The Mann-Whitney U-test revealed that the distribution of lymphocytes and granulocytes was significantly different in seronegative and seropositive cows $(P=0.022)$. In contrast, none of the other parameters showed a significant difference between the considered groups of animals.

The effect of Besnoitia infection on hematological and biochemical parameters, enzyme activities and serum cortisol levels was subsequently considered using GLMs. The interactions between Besnoitia infection, "number of parturitions" and/or "phase of lactation" were also considered to take into account both the influence of the parasite infection and the physiological status of the cows on the considered laboratory parameters. Final models in which the results on the selected parameters were associated with B. besnoiti infection are reported in Table 2; final models in which only the variables "number of parturitions" or "phase of lactation" were statistically associated to the response variable are reported in Additional file 3: Table S3. Only results related to B. besnoiti infection are discussed, since those related merely to the physiological status of the cows are not relevant to this study. The data of each full model with reference to backward selection procedure are summarized in Additional file 4: Tables S4.

Regarding hematological parameters, only the variables lymphocytes and granulocytes were influenced by both $B$. besnoiti seropositivity and clinical disease. The percentage of lymphocytes was evidenced to be lower in seropositive (all values presented as the mean $\pm \mathrm{SD} ; 45.78 \pm$ $18.53 \%$, odds ratio [OR] 0.93) than in seronegative cows $(53.52 \pm 13.69 \%)$. Also, the percentage of granulocytes was 1.08-fold higher in seropositive cattle than in seronegative cattle $(54.22 \pm 18.53$ vs $46.48 \pm 13.69 \%$, respectively). In addition, the interaction between $B$. besnoiti seropositivity and the number of parturitions was statistically associated to the alterations in the leukocyte differential, i.e. the percentage of lymphocytes $(37.81 \pm 16.51$, OR 0.85) was lower and the percentage of granulocytes $(62.19 \pm 16.51 \%$, OR 1.18$)$ was higher in seropositive primiparous cows than in seropositive multiparous cows and their respective seronegative counterparts. Similarly, clinically affected cows evidenced lower percentages of lymphocytes $(47.88 \pm 18.15 \%$, OR 0.86$)$ and higher percentages of granulocytes (52.12 $\pm 18.15 \%$, OR 1.04) when compared to seronegative or asymptomatic seropositive cows ( $51.70 \pm 16.12$ and $48.30 \pm 16.12 \%$, respectively). Also, in this case, the interaction between clinical signs of besnoitiosis and the number of parturitions was associated with lower and higher percentage values of lymphocytes (38.75 $\pm 17.98 \%$, OR 0.86) and granulocytes $(61.25 \pm 17.98 \%$, OR 1.16), respectively, in clinically affected primiparous cows, when compared to multiparous animals with clinical signs of chronic besnoitiosis and seronegative or asymptomatic seropositive animals (Table 2). 


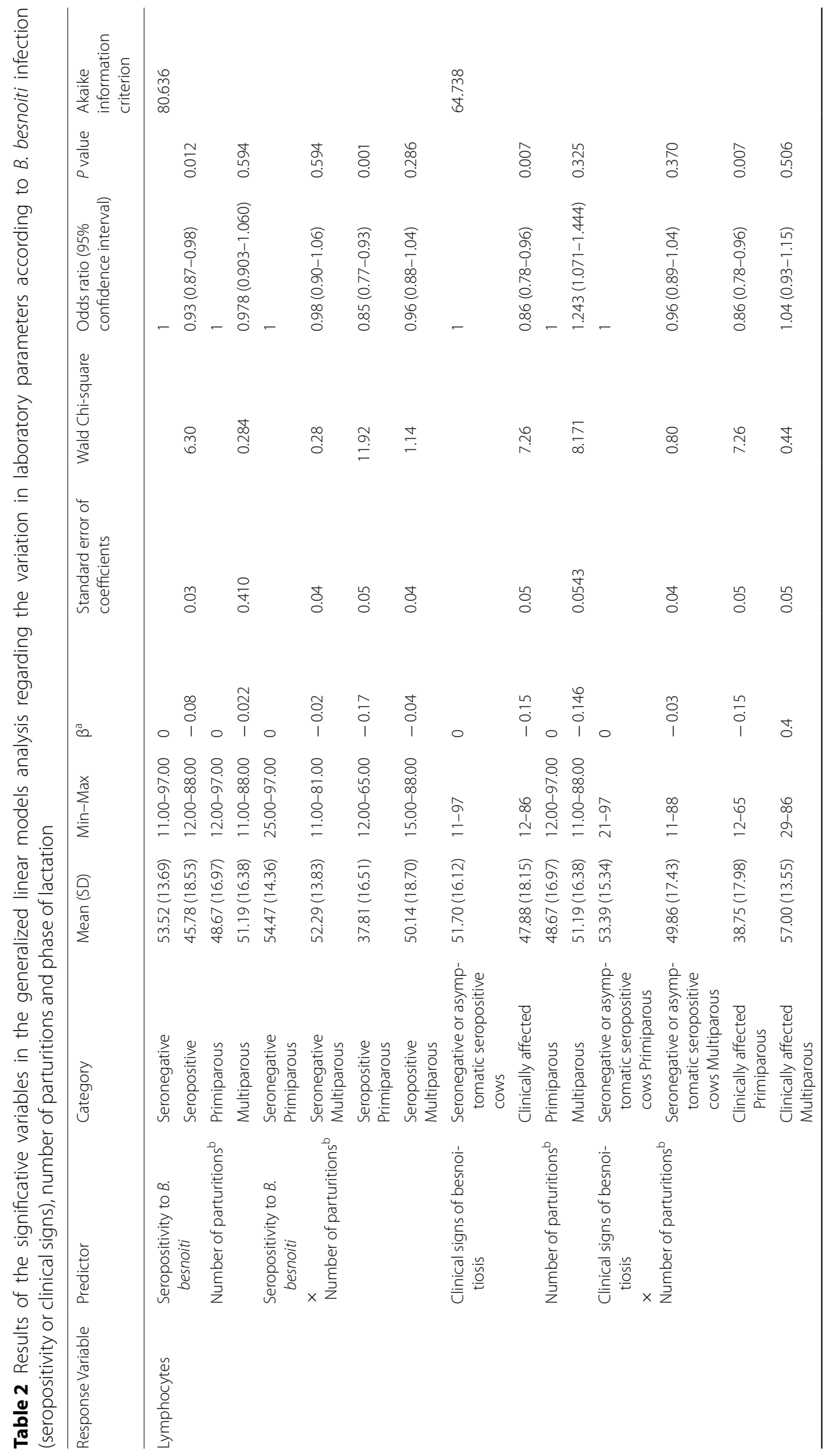




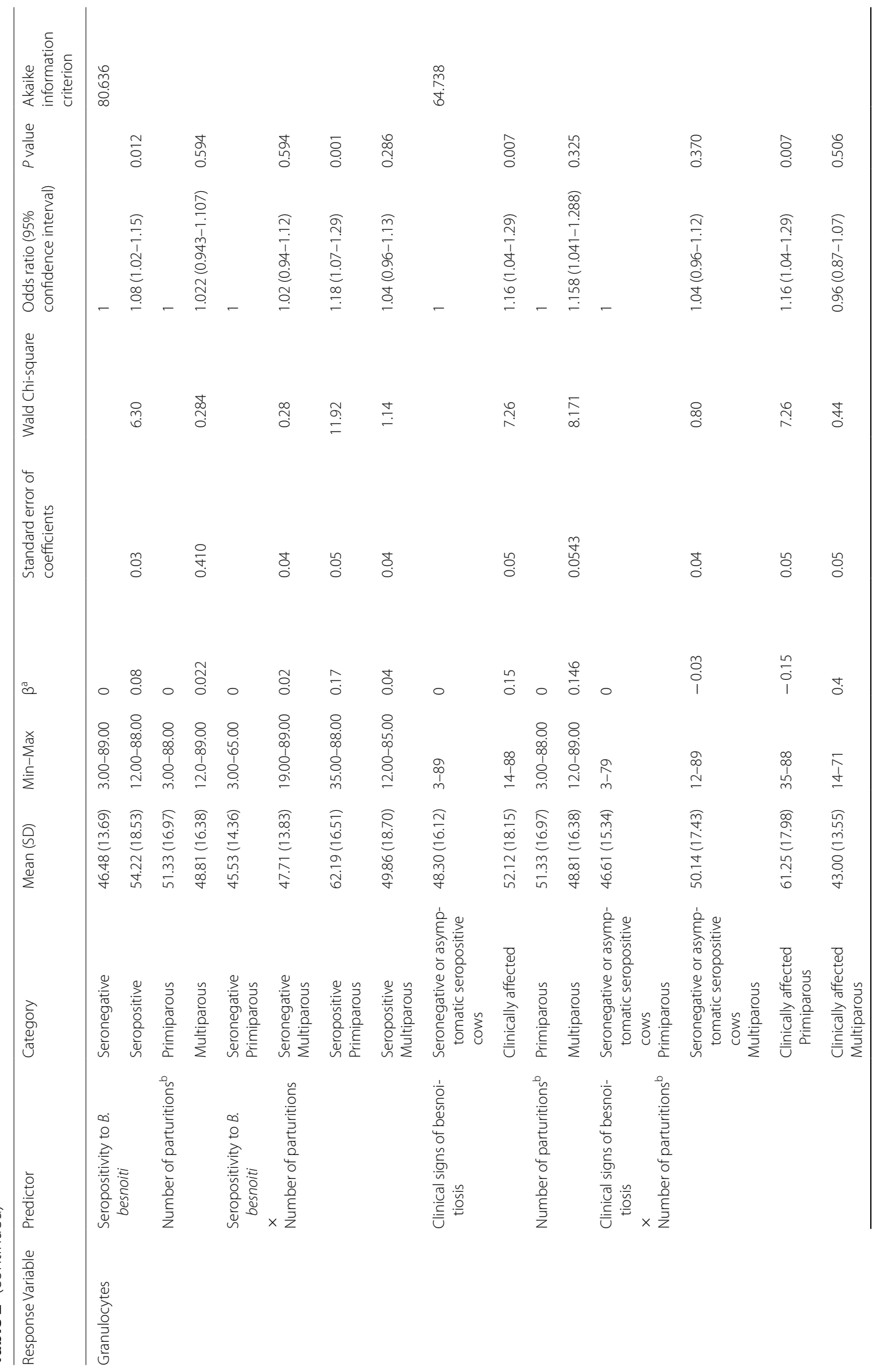




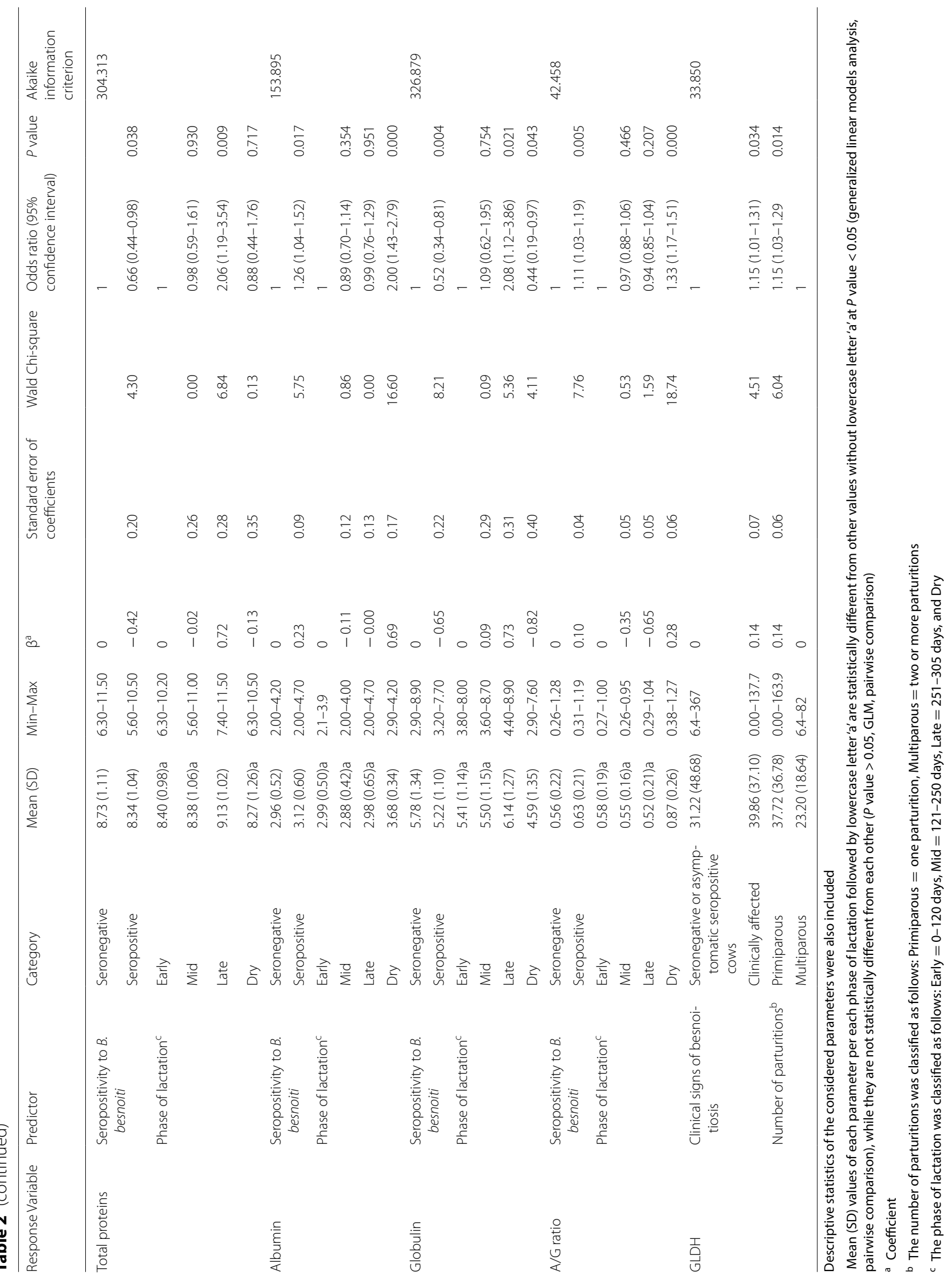


Regarding biochemical analytes, alterations in serum protein values were associated with $B$. besnoiti serological status and the lactation phase. Parasite infection was a predictor of lower values of total protein $(8.34 \pm 1.04 \mathrm{~g} /$ $\mathrm{dl}$, OR 0.66) and globulin $(5.22 \pm 1.10 \mathrm{~g} / \mathrm{dl}$, OR 0.52) in seropositive cows, in comparison to seronegative animals $(8.73 \pm 1.11$ and $5.78 \pm 1.34 \mathrm{~g} / \mathrm{dl}$, respectively). On the contrary, higher values of albumin $(3.12 \pm 0.60 \mathrm{~g} / \mathrm{dl}$, OR 1.26) and A/G ratio $(0.63 \pm 0.21$, OR 1.11$)$ were evidenced in seropositive cows compared to seronegative ones $(2.96 \pm 0.52$ $\mathrm{g} / \mathrm{dl}$ and $0.56 \pm 0.22$, respectively). Regarding the lactation phase, higher total protein values $(9.13 \pm 1.02 \mathrm{~g} /$ $\mathrm{dl}$, OR 2.06) were evidenced in cows in the late phase of lactation, whereas dry cows showed a higher probability to have higher values for both albumin $(3.68 \pm 0.34 \mathrm{~g} / \mathrm{dl}$, OR 2.00) and A/G ratio ( $0.87 \pm 0.26$, OR 1.33). Furthermore, cows in the late phase of lactation and dry animals had a higher probability to show higher and lower globulin values $(6.15 \pm 1.27$, OR 2.08 and $4.59 \pm 1.35 \mathrm{~g} / \mathrm{dl}$, OR 0.44 , respectively) than cows of other lactation categories (Table 2).

Among the enzyme activity parameters, the presence of clinical signs of besnoitiosis and the number of parturitions were associated to alterations in GLDH values. Indeed, clinically affected animals had an enhanced probability to have higher mean values of GLDH $(39.86 \pm 37.10 \mathrm{U} / \mathrm{l}$, OR 1.15) when compared to seronegative or asymptomatic seropositive cows $(31.22 \pm 48.68 \mathrm{U} / \mathrm{l})$. Similarly, primiparous cows (37.72 $\pm 36.78 \mathrm{U} / \mathrm{l}$, OR 1.15$)$ had an enhanced probability to have higher GLDH values than multiparous animals $(23.20 \pm 18.64 \mathrm{U} / \mathrm{l})$.

\section{Discussion}

The study provides data on laboratory parameters, including hematology, biochemistry, enzyme activity and cortisol determination, in B. besnoiti naturally infected cows from an endemically affected dairy cattle herd in Italy. Results were analyzed taking into consideration both the serological status for $B$. besnoiti (seronegative and seropositive cows) and the presence of clinical chronic bovine besnoitiosis (seronegative or asymptomatic seropositive cows and clinically affected cows). The influence of the number of parturitions (primiparous and multiparous cows) and the phase of lactation (early, mid, late and dry) on laboratory parameters were also considered. Finally, the two-way interactions between Besnoitia seropositivity or clinical disease, number of parturitions and phase of lactation were evaluated.

\section{Hematology}

Mean RBC counts were at the lower limit of the reference range in all sampled animals. However, it should be noted that of the 107 cows sampled for blood parameters,
95 were lactating at the time of sampling, and lactating cows normally consistently have lower RBC counts than non-lactating cows [20]. A few authors demonstrated the occurrence of anemia in both beef cattle [9] and goats [13] with besnoitiosis, probably caused by the chronic inflammatory state.

An important alteration was evidenced in WBC counts. Even though the mean number of WBC fell within the normal range in all considered groups of animals, $B$. besnoiti infection caused an alteration in the leukocyte differential, with a higher percentage of granulocytes and a lower percentage of lymphocytes in seropositive and clinically affected cows. The same alteration was influenced by the number of parturitions, and was enhanced for seropositive and clinically affected animals in the group of primiparous cows. Many seropositive animals (69.6\%), particularly primiparous cows (87.5\%), presented this alteration in the leukocyte differential. Indeed, a significative interaction was evidenced between parasite infection and physiological status of the cows: this alteration in the leukocyte differential, with higher values of granulocytes and lower values of lymphocytes, was influenced not only by $B$. besnoiti seropositivity or clinical disease, but also by the physiological status of the cows. This finding suggests that the effects of the parasite infection may be enhanced in primiparous cows if compared to multiparous cows, probably due to a higher level of stress and immunosuppression in younger animals.

Langenmayer et al. [9] reported leukopenia in the acute phase of the disease followed by higher WBC concentration values after seroconversion in beef cattle. Two other studies evidenced leukocytosis in cattle infected with $B$. besnoiti: Dubey et al. [11] also reported a left-shift neutrophilia in a clinically affected bull, whereas Nieto-Rodríguez et al. [10] detected lymphocytosis in some infected beef animals. Nazifi et al. [13] evidenced that, compared to normal goats, WBC and neutrophils were significantly higher and lymphocytes were significantly lower in goats with clinical besnoitiosis.

It is recognized that in adult cattle lymphocytes remain the dominant cell type, with a neutrophil-to-lymphocyte ratio of approximately 1:2. A shift in this ratio (stress leukogram) may be due to several causes: inflammatory granulocytosis is reported in viral, bacterial, protozoal, parasitic and fungal infections, whereas stress, infections, immune suppression, also related to corticosteroids, are among the causes of lymphopenia [21]. Besides, the aggregation of lymphocytes around Besnoitia cysts in affected organs could also induce lymphopenia [13].

In this context, it should be emphasized that the farm is officially free of tuberculosis, brucellosis and leucosis. All of the animals in the herd are vaccinated against Bovine Viral Diarrhea Virus, and the farm adheres to the 
management plan for the control of infectious bovine rhinotracheitis and paratuberculosis. Further, after the diagnosis of some cases of $\mathrm{Q}$ fever also causing abortions in the herd 1 year earlier, all the animals were vaccinated against Coxiella burnetii. Moreover, it is noteworthy to emphasize that a form of generalized demodectic mange in two dairy cows co-infected with $B$. besnoiti was diagnosed and that Demodex bovis mites could also affect other animals, causing subclinical forms of the disease and possibly contributing to the detected alterations in WBC parameters [19]. Therefore, the observed alteration in the leukocyte differential could be a predictor of the parasite infection, suggesting the need to include bovine besnoitiosis in the differential diagnoses along with other diseases.

Any relevant alteration was not detected in platelet indexes, with only a slight reduction in the mean values of PLT counts, MPV, Pct and PDW in seropositive and clinically affected animals, suggesting that slight thrombocytopenia may indicate the presence of an inflammatory response. It is possible that the increase in platelet consumption for their destruction during the chronic inflammatory process due to the parasite infection may be the factors determining these findings [22]. However, considering that none of the previous studies investigated these parameters, it is not possible to draw significant conclusions.

\section{Biochemistry and enzyme activity}

Serum total proteins were elevated in all cows, with a slightly higher value in seronegative animals; the parasite infection caused lower mean values of total proteins in seropositive animals. Also, a higher level of albumin and a lower level of globulin were associated with $B$. besnoiti infection, as detected in seropositive cows. Langenmayer et al. [9] reported higher values of total proteins, albumin and globulin in seropositive cattle, and some other animals with clinical besnoitiosis evidenced high levels of total proteins and albumin [10]; similar findings were also shown in clinically infected goats [14]. Moderate hypoalbuminemia and mild hyperglobulinemia were reported during the acute phase in a clinically affected bull [11]. However, it should be considered that these results refer to studies performed on beef cattle. Alshehabat et al. [12] reported a slightly lower value in total proteins with a higher value of albumin in B. besnoiti infected dairy cows.

Regarding total proteins, it can not be excluded that seropositive cows may manifest edematous phenomena associated with hypoproteinemia conditions that are not detectable at the clinical examination due to the infection in these animals being subclinical. Higher mean values of albumin in seropositive animals could indicate slight dehydration; instead, clinically affected animals showed a slightly lower albumin concentration when compared to the other groups of cows, probably due to inflammation (as albumin acts as a negative acute phase protein) and negative energy balance. In any case, mean values of albumin were at the lower limit of the range for all animals. The lower values of globulin in seropositive animals, even with elevated mean values in all the animals, could be due to immunosuppression, taking into consideration the higher levels of serum cortisol and the lower values of lymphocytes [23]. Moreover, it was evidenced that also the phase of lactation influenced serum protein parameters, reflecting the physiological changes occurring during the lactation period.

The higher values of total bilirubin, particularly in seropositive and clinically affected cows, evidenced also in beef cattle by Langenmayer et al. [9], could be related to both prehepatic icterus due to hemorrhages, and anorexia, sickness, and debilitation.

No alterations were detected in mean $\mathrm{Ca}, \mathrm{P}$ and $\mathrm{Mg}$ concentrations, with all values falling within the reference ranges and no difference between seronegative and seropositive cows. However, it should be considered that blood concentrations of minerals are influenced by physiological factors, such as food intake and hydration, and can also change daily [9].

Concerning metabolites and ketones, no difference was underlined between seronegative, seropositive and clinically affected cows. However, if considering all the animals, $\mathrm{BOHB}$ and lactate were both slightly higher, indicating a reduced energy balance in milk-producing dairy cows, whereas NEFA values fell within the reference range.

Regarding enzyme activities, no relevant alteration was detected for AST, CK and LDH in B. besnoiti seropositive and clinically affected animals. Inconsistent results were also reported in previous studies $[9,12]$. AST values were similar in all the considered groups of animals; CK showed a higher activity in seronegative animals, but it should be considered that some animals had very high values, distorting the mean results. According to Langenmayer et al. [9], the effect of tissue cysts on muscle fibers can be very low, leading to only slight and undetectable release of muscle enzymes. LDH mean value was slightly higher in clinically affected animals compared to seronegative ones; this alteration could be associated with tissue damage. GLDH was recorded at levels that were slightly higher than the upper limit of the reference range in all animals, with a higher mean value in clinically affected animals and, particularly, in primiparous cows; these findings could be due to the metabolic condition of the cows. 


\section{Cortisol determination}

Higher cortisol mean values were detected in seropositive and clinically affected cows when compared to seronegative animals, although the difference was not statistically significant.

Mormède et al. [15] reported that mean baseline cortisol values in cattle are typically lower than $10 \mathrm{ng} / \mathrm{ml}$, with recent studies often reporting values of $<5 \mathrm{ng} / \mathrm{mL}$. In the present study, the mean values of cortisol were quite high for all the herd animals: this finding could be related to poor farm management practices that were evidenced during the visits to the farm. Although statistical analysis did not show any relation between $B$. besnoiti infection and cortisol levels, it is hypothesized that, among other physiological, pathological and farm-related factors, higher values of cortisol in B. besnoiti seropositive and clinically affected animals may also be due to the stress related to the disease; indeed, bovine besnoitiosis is a chronic and debilitating disease, compromising animal welfare. In the same farm, a decrease in certain productive parameters (i.e. daily kilograms of milk produced, percentage of fat and protein in the milk and mature equivalent milk yield) was previously evidenced in cows showing tissue cysts in the skin, which could be correlated to the debilitation caused by the chronic phase of the disease [5]. The detection of D. bovis infection in two cows, seropositive for $B$. besnoiti, provides support for this hypothesis: it is known [19] that these mites develop a heavy infection mainly in dairy cows suffering from stress, and that the occurrence of bovine demodicosis seems to be associated with debilitating factors or receptive physiological states of the animal, such as pregnancy or lactation. Since bovine besnoitiosis is a chronic and debilitating disease, its role in determining an immune imbalance, possibly leading to other infections, including $D$. bovis [19], should not be neglected. Stress could be a consequence of $B$. besnoiti infection, but it has also been experimentally demonstrated that immunodepression related to corticosteroids could facilitate parasite proliferation [17]. To the best of the authors' knowledge, this is the first study investigating cortisol levels in B. besnoiti seropositive and clinically affected cattle. Although the results are only preliminary, it is suggested that $B$. besnoiti may cause a higher value of cortisol and then stress in infected animals: the consequences for animal welfare should not be neglected. Besides, the effect of cortisol on both the disease onset and progression should be considered. However, also considering the lack of statistical significance, the association between $B$. besnoiti infection and cortisol levels could not be demonstrated, and this hypothesis should be further investigated.

\section{Conclusions}

The study provides data on alterations in laboratory parameters in $B$. besnoiti seropositive and clinically affected cows from an endemically infected dairy herd in Italy. Only mild changes were found in hematology, biochemistry, and enzyme activity parameters. Besnoitia besnoiti infection seemed to be associated with an alteration in the leukocyte differential, characterized by a higher percentage of the granulocytes and a lower value of the lymphocytes population, particularly in primiparous cows. Considering biochemistry and enzyme activity parameters, the parasite infection caused alterations in serum protein parameters in seropositive animals and in GLDH values in clinically affected ones. Although not statistically significant, the higher cortisol levels in seropositive and clinically affected animals may suggest that bovine besnoitiosis could be related to stress in infected cows: this may lead to consequences not only on animal welfare but also on the disease onset and progression. However, laboratory values are influenced by both physiological and pathological factors. Besides, it should be considered that farm-related factors could influence results on investigated animals. Further studies involving a higher number of animals from different farms, including seropositive cattle and clinically affected cows in both acute and chronic phase of the disease, could help to determine the role of $B$. besnoiti in alterations in laboratory parameters, and to aid veterinarians in the diagnosis of bovine besnoitiosis to optimize a control plan for the disease in the infected herds.

\section{Supplementary Information}

The online version contains supplementary material available at https://doi. org/10.1186/s13071-021-04626-4.

Additional file 1: Table S1. Descriptive statistics (mean, standard deviation, minimum and maximum) of hematological, biochemical and enzyme activities analyses, and cortisol determination sorted by the number of parturitions and the serological and clinical status of cows in a dairy cattle herd endemically infected by bovine besnoitiosis.

Additional file 2: Table S2. Descriptive statistics (mean, standard deviation, minimum and maximum) of hematological, biochemical and enzyme activities analyses, and cortisol determination sorted by the lactation phase and the serological and clinical status of cows in a dairy cattle herd endemically infected by bovine besnoitiosis.

Additional file 3: Table S3. Results of the significative variables in the GLM analysis regarding the variation on laboratory parameters according to number of parturitions and phase of lactation. Descriptive statistics (mean, standard deviation, minimum and maximum) of the considered parameters were also included.

Additional file 4: Tables S4. Selection of final model by backward elimination according to the best Akaike's information criterion (AIC). AIC values were provided for the full models and for each step corresponding to the removal of the least statistically significant variable, until the final model was obtained. 


\section{Acknowledgements \\ Not applicable.}

\section{Authors' contributions}

LV and MTM conceived and designed the study. LV and ALG collected the samples. LV, ALG, SAZ, SM and AG performed the laboratorial analyses. LV and ALG performed the statistical analysis. LV, ALG and MTM wrote the manuscript. All authors read and approved the final manuscript.

\section{Funding}

This research did not receive any specific grant from funding agencies in the public, commercial, or not-for-profit sectors.

\section{Availability of data and materials}

The datasets used and/or analyzed during the current study are available from the corresponding author on reasonable request.

\section{Ethics approval and consent to participate}

Biological samples collection was performed by qualified veterinarians applying adequate procedures of handling and disinfection to minimize pain or distress in sampled animals. All procedures for the collection of biological specimens from live animals were accomplished following good clinical practices in the respect of animal welfare according to all applicable international, national, and institutional guidelines for the care and use of animals. The study was conducted with the approval of the Institutional Animal Care and Use Committee of Università degli Studi di Milano (Prot. no OPBA_34_2017).

\section{Consent for publication}

Not applicable.

\section{Competing interests}

The authors declare that they have no competing interests.

Received: 12 August 2020 Accepted: 6 February 2021

Published online: 15 March 2021

\section{References}

1. European Food Safety Authority (EFSA). Bovine besnoitiosis: an emerging disease in Europe. EFSA J. 2010;8:1499-514.

2. Gentile A, Militerno G, Schares G, Nanni A, Testoni S, Bassi P, et al. Evidence for bovine besnoitiosis being endemic in Italy: first in vitro isolation of Besnoitia besnoiti from cattle born in Italy. Vet Parasitol. 2012;184:108-15.

3. Gazzonis AL, Alvarez-Garcia G, Zanzani SA, Garippa G, Rossi L, Maggiora $\mathrm{M}$, et al. Besnoitia besnoiti among cattle in insular and northwestern Italy: endemic infection or isolated outbreaks? Parasites Vectors. 2014;7:585.

4. Gazzonis AL, Alvarez-Garcia G, Maggioni A, Zanzani SA, Olivieri E, Compiani R, et al. Serological dynamics and risk factors of Besnoitia besnoiti infection in breeding bulls from an endemically infected purebred beef herd. Parasitol Res. 2017;116:1383-93.

5. Villa L, Gazzonis AL, Zanzani SA, Perlotti C, Sironi G, Manfredi MT. Bovine besnoitiosis in an endemically infected dairy cattle herd in Italy: serological and clinical observations, risk factors, and effects on reproductive and productive performances. Parasitol Res. 2019;118:3459-68.

6. Alvarez-Garcia G, Frey CF, Ortega-Mora LM, Schares G. A century of bovine besnoitiosis: an unknown disease re-emerging in Europe. Trends Parasitol. 2013;29:407-15.
7. Cortes H, Leitao A, Gottstein B, Hemphill A. A review on bovine besnoitiosis: a disease with economic impact in herd health management, caused by Besnoitia besnoiti. Parasitology. 2014;141:1406-17.

8. Jacquiet P, Liénard E, Franc M. Bovine besnoitiosis: epidemiological and clinical aspects. Vet Parasitol. 2010;174:30-6.

9. Langenmayer MC, Scharr JC, Sauter-Louis C, Schares G, Gollnick NS. Natural Besnoitia besnoiti infections in cattle: hematological alterations and changes in serum chemistry and enzyme activities. BMC Vet Res. 2015;11:32.

10. Nieto-Rodríguez JM, Calero-Bernal R, Álvarez-García G, Gutiérrez-Expósito D, Redondo-García E, Fernández-García JL, et al. Characterization of an outbreak of emerging bovine besnoitiosis in southwestern Spain. Parasitol Res. 2016;115:2887-92.

11. Dubey JP, Van Wilpe E, Blignaut DJC, Schares G, Williams JH. Development of early tissue cysts and associated pathology of Besnoitia besnoiti in a naturally infected bull (Bos taurus) from South Africa. J Parasitol. 2013;99:459-66.

12. Alshehabat $M$, Alekish M, Talafha A. Selected metabolic biochemical and enzyme activities associated with Besnoitia besnoiti infection in dairy cattle. Trop Anim Health Prod. 2016:48:1301-4.

13. Nazifi S, Oryan A, Mohebbi H. Evaluation of hematological parameters in caprine besnoitiosis. J Appl Anim Res. 2002;21:123-8.

14. Oryan A, Nazifi S, Mohebbi H. Pathology and serum biochemical changes in natural caprine besnoitiosis. Rev Med Vet. 2008;159:27-32.

15. Mormède $P$, Andanson S, Aupérin B, Beerda B, Guémené D, Malmkvist J, et al. Exploration of the hypothalamic-pituitary-adrenal function as a tool to evaluate animal welfare. Physiol Behav. 2007:92:317-39.

16. Brown EJ, Vosloo A. The involvement of the hypothalamo-pituitaryadrenocortical axis in stress physiology and its significance in the assessment of animal welfare in cattle. Onderstepoort J Vet Res. 2017;84:a1398.

17. Álvarez-García G, García-Lunar P, Gutiérrez-Expósito D, Shkap V, OrtegaMora LM. Dynamics of Besnoitia besnoiti infection in cattle. Parasitology. 2014;141:1419-35.

18. Gutiérrez-Expósito D, Ferre I, Ortega-Mora LM, Álvarez-García G. Advances in the diagnosis of bovine besnoitiosis: current options and applications for control. Int J Parasitol. 2017:47:737-51.

19. Villa L, Gazzonis AL, Perlotti C, Zanzani SA, Sironi G, Manfredi MT. First report of Demodex bovis infestation in bovine besnoitiosis co-infected dairy cattle in Italy. Parasitol Int. 2020;75:102021.

20. Wood D, Quiroz-Rocha GF. Normal hematology of cattle. In: Weiss DJ, Wardrop KJ, editors. Schalm's veterinary hematology. Ames: Wiley; 2010. p. 829-35.

21. Roland L, Drillich M, Iwersen M. Hematology as a diagnostic tool in bovine medicine. J Vet Diagn Invest. 2014;26:592-8.

22. Russell KE. Platelet kinetics and laboratory evaluation of thrombocytopenia. In: Weiss DJ, Wardrop KJ, editors. Schalm's veterinary hematology. Ames: Wiley; 2010. p. 576-85

23. Stockham SL, Scott MA. Proteins. In: Stockham SL, Scott MA, editors. Fundamentals of veterinary clinical pathology. Ames: Blackwell Publishing: 2008. p. 369-414

\section{Publisher's Note}

Springer Nature remains neutral with regard to jurisdictional claims in published maps and institutional affiliations. 